\title{
Fermion portal dark matter
}

\author{
Yang Bai ${ }^{a}$ and Joshua Berger ${ }^{b}$ \\ ${ }^{a}$ Department of Physics, University of Wisconsin, \\ 1150 University Avenue, Madison, WI 53706, U.S.A. \\ ${ }^{b}$ SLAC National Accelerator Laboratory, \\ 2575 Sand Hill Road, Menlo Park, CA 94025, U.S.A. \\ E-mail: yangbai@physics.wisc.edu, jberger@slac.stanford.edu
}

\begin{abstract}
We study a class of simplified dark matter models in which one dark matter particle couples with a mediator and a Standard Model fermion. In such models, collider and direct detection searches probe complimentary regions of parameter space. For Majorana dark matter, direct detection covers the region near mediator-dark matter degeneracy, while colliders probe regions with a large dark matter and mediator mass splitting. For Dirac and complex dark matter, direct detection is effective for the entire region above the mass threshold, but colliders provide a strong bound for dark matter lighter than a few $\mathrm{GeV}$. We also point out that dedicated searches for signatures with two jets or a monojet not coming from initial state radiation, along missing transverse energy can cover the remaining parameter space for thermal relic dark matter.
\end{abstract}

KeYwords: Beyond Standard Model, Cosmology of Theories beyond the SM

ARXIV EPRINT: 1308.0612 


\section{Contents}

1 Introduction 1

2 Simplified dark matter model: fermion portal 3

3 Relic abundance $\quad 4$

4 Dark matter direct detection $\quad 6$

5 Collider constraints $\quad 8$

5.1 Estimated limits from monojet on $t$-change $\phi$ exchange 8

5.2 Limits from $2 j+E_{T}^{\text {miss }}$ on $\phi$ pair production 9

$\begin{array}{lll}5.3 & \text { Limits from monojet on single } \phi \text { productions } & 10\end{array}$

6 Discussion and conclusions 13

\section{Introduction}

There is now a large body of evidence for the existence of particulate dark matter that interacts gravitationally and makes up nearly a quarter of the energy density of the universe [1-4]. On the other hand, such dark matter does not exist in the Standard Model (SM), has not been conclusively observed to interact non-gravitationally, and has never been detected, directly or indirectly, in a laboratory experiment. Determining the physical properties of dark matter therefore constitutes one of the most pressing questions in highenergy physics. Ideally, it would be possible to measure the non-gravitational interactions in several ways: by directly detecting rare ambient dark matter scattering events off a target in underground laboratories, by detecting the products of dark matter self-annihilation or decay, and by measuring a momentum imbalance in collider events.

Given that so little is known about the properties of dark matter, the most informative framework for studying dark matter properties in a simplified framework is as modelindependent as possible. One powerful approach is to consider effective operators of dark matter coupling to quarks and other SM particles [5-25]. In the absence a light mediator below around one $\mathrm{GeV}$, the effective operator approach provides an excellent description for interpreting the direct detection experiment results because of the small exchanged momentum in the scattering processes. A simplified description in terms of such operators neatly encapsulates all possible interactions of dark matter with their detectors. On the other hand, such operators may be unsuitable or at least not precise for the purposes of collider studies [26-28]. The latest searches for dark matter at ATLAS [21, 29] and CMS [25, $30]$ have set a lower bound on the cutoffs of the effective operators to be around one $\mathrm{TeV}$. The current constraints are stringent when compared to the limits derived from direct 
detection experiments, but are still much below the center-of-mass energy, $8 \mathrm{TeV}$, of the latest run at the LHC. When the parton-level collision energy is comparable to the mediator mass, the effective theory does not apply and a more complete description is required.

To go beyond an effective operator approach, one could directly use a concrete underlying model such as the Minimal Supersymmetric Standard Model (MSSM) to study the complementarity among different experimental probes of dark matter [31]. However, to maintain the model-independent paradigm of the effective operator approach, a "Simplified Dark Matter Model" could be a better choice. In this approach, one can introduce one or two new particles with one or two interactions to probe the potentially complicated dark matter sector. For dark matter interacting with SM particles, one of the most important things is the properties of the mediator. The majority of existing studies in the literature have the mediator particle couple to two dark matter particles at the same time: Higgs portal [32-34], 2HDM portal [35], axion-portal [36], gravity-mediated [37], dilaton-assisted [38] and $Z^{\prime}$-mediated $[39,40]^{1}[43]$. In this paper, we study simplified dark matter models with SM fermions as the portal particle, which we call Fermion Portal (FP) dark matter. This class of models is well motivated and can easily be a part of some underlying models such as SUSY [44] or extra-dimension models [45].

In FP dark matter models, an SM singlet dark matter particle interacts with quarks via a new QCD color triplet state. There are several classes of such models depending on the Lorentz properties of the dark matter: it may be a Dirac fermion, Majorana fermion, complex scalar, real scalar, or vector. The vector case requires an additional scalar field or additional dynamics to provide the vector boson mass and we do not consider it here. For the real scalar dark matter case, the non-relativistic interaction cross-sections including self-annihilation and direct detection are highly suppressed and we do not consider this case either. Therefore, in this paper we perform detailed studies of the Dirac fermion, Majorana fermion, and complex scalar cases.

A further specification can be made based on species of quarks to which the dark matter couples. At renormalizable level, the simplest interactions are to have dark matter particles couple to only right-handed fermions. In the non-relativistic limit, the interactions can be transformed as vector or axi-vector couplings between the dark matter particle and the SM fermion. For either type of couplings, a dark matter particle that couples exclusively to heavy quarks would have a suppressed direct detection cross-section. We concentrate on the couplings to up and down quarks in this paper and leave the heavy quark case for future exploration.

The six cases that are considered here constitute a set of models for benchmarking the progress of dark matter experiments, as well as for studying the complementarity of different types of experiments. Unlike the effective operator approach, they can be extrapolated to arbitrarily high energies while giving sensible results. Furthermore, they are well-motivated and simple enough to allow for deep experimental scrutiny. In this paper, we demonstrate the power of current experiments to probe these models and determine the

\footnotetext{
${ }^{1}$ The milli-charged dark matter with only one massless gauge boson in this model contradicts quantum gravity, as shown in ref. [41] based on arguments in ref. [42].
} 
allowed parameter space to probe in the future. For each case, we also show the parameter space to satisfy the dark matter thermal relic abundance. We pay attention to the potential for current experiments to probe the thermal relic hypothesis. We find that the parameter space for a thermal relic is highly constrained for most of the scenarios considered, but that there is some potential at the moment in a model with Majorana dark matter.

The remainder of this paper is structured as follows. In section 2, we introduce the Fermion Portal class of simplified models. We determine the allowed parameter space for dark matter to be a thermal relic in section 3. Current direct detection and collider constraints are determined in sections 4 and 5 respectively, with summary plots presented in section 5. We discuss potential improvement for the LHC collider searches and conclude in section 6 .

\section{Simplified dark matter model: fermion portal}

If the dark matter sector interacts directly with a single fermion in the SM, two particles with different spins are required in the dark matter sector. In this paper, we will concentrate on the quark portal dark matter and leave the lepton portal dark matter for future exploration. Restricting to particles with a spin less than one, there are two general situations: fermionic dark matter with a color-triplet scalar partner or scalar dark matter with a color-triplet fermion partner. In the former case, we consider both Dirac and Majorana dark matter, while for the latter case we only consider a complex scalar dark matter and skip the real scalar dark matter case [6], which has a quark mass suppressed $s$-wave or a $d$ wave or three-body suppressed annihilation rate and a velocity suppressed direct detection cross section if the quark masses are neglected.

We begin by considering fermionic dark matter coupled to right-handed quarks as the portal to the dark matter sector. The dark matter candidate may be a Dirac or Majorana fermion, $\chi$, that is an $\mathrm{SM}$ gauge singlet. The mediator is an $\mathrm{SU}(3)_{c}$ triplet with an appropriately chosen hypercharge. The renormalizable operators are

$$
\mathcal{L}_{\text {fermion }} \supset \lambda_{u_{i}} \phi_{u_{i}} \bar{\chi}_{L} u_{R}^{i}+\lambda_{d_{i}} \phi_{d_{i}} \bar{\chi}_{L} d_{R}^{i}+\text { h.c. },
$$

where $u_{i}=u, c, t\left(d_{i}=d, s, b\right)$ are different SM quarks. Since $\chi$ is the dark matter candidate, the partner masses $m_{\phi_{i}}$ must be larger than the dark matter mass $m_{\chi}$. In our analysis, we assume the branching ratio of the decay $\phi_{u_{i}} \rightarrow \chi \bar{u}^{i}$ and $\phi_{d_{i}} \rightarrow \chi \bar{d}^{i}$ is $100 \%$. We also require the Yukawa couplings $\lambda_{i}$ to be less than $\sqrt{4 \pi}$ to preserve perturbativity. Since we will concentrate on the first generation quarks, we neglect the flavor index from now on to simplify the notation. Using the up quark operator, the width of $\phi_{u}$ particle is calculated to be

$$
\Gamma(\phi \rightarrow \chi+\bar{u})=\frac{\lambda_{u}^{2}}{16 \pi} \frac{\left(m_{\phi}^{2}-m_{\chi}^{2}\right)^{2}}{m_{\phi}^{3}},
$$

for both Dirac and Majorana cases.

Similarly, for a complex scalar dark matter, $X$, and its partner, $\psi$, a color-triplet Dirac fermion, we have the interactions

$$
\mathcal{L}_{\text {scalar }} \supset \lambda_{u_{i}} X \bar{\psi}_{L}^{u_{i}} u_{R}^{i}+\lambda_{d_{i}} X \bar{\psi}_{L}^{d_{i}} d_{R}^{i}+\text { h.c. } .
$$


For the up quark operator, the decay width of $\psi^{u}$ field is

$$
\Gamma\left(\psi \rightarrow X^{\dagger}+u\right)=\frac{\lambda_{u}^{2}}{32 \pi} \frac{\left(m_{\psi}^{2}-m_{X}^{2}\right)^{2}}{m_{\psi}^{3}} .
$$

If the operators in eqs. (2.1), (2.3) are defined in the flavor basis, the quark righthanded currents become physical and additional (weak) flavor constraints apply to the model parameter space. However, if they are defined in the quark mass basis, there are no additional flavor changing processes beyond the SM. We simply take the mass basis assumption and ignore the flavor physics constraints. We next explore the dark matter phenomenology of this class of models, including its thermal relic abundance, direct detection and collider searches. Some other studies for the spin-dependent direct detection and indirect detection signatures can be found in refs. [46, 47].

\section{Relic abundance}

The complimentarity between dark matter collider and direct detection searches is independent of the dark matter thermal history. Since the weakly interacting massive particle (WIMP) is still the best motivated scenario that generates the observed dark matter relic abundance for a weak-scale mass, we calculate the thermal relic abundance for the simplified fermion-portal dark matter. We then compare the allowed thermal relic parameter space to direct detection and collider bounds.

In the fermionic dark matter case, the main annihilation channel is $\bar{\chi} \chi \rightarrow \bar{u} u$ for Dirac dark matter. The dominant contribution to the annihilation cross-section is

$$
\frac{1}{2}(\sigma v)_{\text {Dirac }}^{\chi \bar{\chi}}=\frac{1}{2}\left[\frac{3 \lambda_{u}^{4} m_{\chi}^{2}}{32 \pi\left(m_{\chi}^{2}+m_{\phi}^{2}\right)^{2}}+v^{2} \frac{\lambda_{u}^{4} m_{\chi}^{2}\left(-5 m_{\chi}^{4}-18 m_{\chi}^{2} m_{\phi}^{2}+11 m_{\phi}^{4}\right)}{256 \pi\left(m_{\chi}^{2}+m_{\phi}^{2}\right)^{4}}\right] \equiv s+p v^{2},
$$

where $v$ is the relative velocity of two dark matter particles and is typically $0.3 c$ at the freeze-out temperature and $10^{-3} c$ at present. The factor of $1 / 2$ in eq. (3.1) accounts for the fact that Dirac dark matter is composed of both a particle and an anti-particle. For Majorana dark matter, the annihilation rate only contains a $p$-wave contribution at leading order in the limit of zero quark masses

$$
(\sigma v)_{\text {Majorana }}^{\chi \chi}=v^{2} \frac{\lambda_{u}^{4} m_{\chi}^{2}\left(m_{\chi}^{4}+m_{\phi}^{4}\right)}{16 \pi\left(m_{\chi}^{2}+m_{\phi}^{2}\right)^{4}} \equiv p v^{2} .
$$

In the non-degenerate parameter space, we only need to care about the dark matter annihilation rate. The dark matter relic abundance is approximately related to the " $s$ " and " $p$ " variables by

$$
\Omega_{\chi} h^{2} \approx \frac{1.07 \times 10^{9}}{\mathrm{GeV} M_{\mathrm{Pl}} \sqrt{g^{*}}} \frac{x_{F}}{s+3(p-s / 4) / x_{F}},
$$

where the Planck scale is $M_{\mathrm{Pl}}=1.22 \times 10^{19} \mathrm{GeV}$ and $g^{*}$ is the number of relativistic degrees of freedom at the freeze-out temperature and is taken to be 86.25 here. The freeze-out 
temperature $x_{F}$ is given by

$$
x_{F}=\ln \left[\frac{5}{4} \sqrt{\frac{45}{8}} \frac{g}{2 \pi^{3}} \frac{M_{\mathrm{Pl}} m_{\chi}\left(s+6 p / x_{F}\right)}{\sqrt{g^{*}} \sqrt{x_{F}}}\right],
$$

where $g=2(4)$ is the number of degrees of freedom for the Majorana (Dirac) fermion dark matter.

In the degenerate parameter space with $\Delta \equiv\left(m_{\phi}-m_{\chi}\right) / m_{\chi} \ll 1$ and comparable to or below the freeze-out temperature $1 / x_{F} \sim 5 \%$, co-annihilation effects become important [48, 49]. Neglecting the sub-leading electroweak interaction, the annihilation cross-section for $\chi+\phi^{\dagger} \rightarrow u+g$ is given by

$$
(\sigma v)^{\chi \phi^{\dagger}}=\frac{g_{s}^{2} \lambda_{u}^{2}}{24 \pi m_{\phi}\left(m_{\chi}+m_{\phi}\right)}+v^{2} \frac{g_{s}^{2} \lambda_{u}^{2}\left(29 m_{\chi}^{2}-50 m_{\chi} m_{\phi}+9 m_{\phi}^{2}\right)}{576 \pi m_{\phi}\left(m_{\chi}+m_{\phi}\right)^{3}},
$$

for both Dirac and Majorana dark matter. Additionally, the $\phi$ field self-annihilation crosssection is given by

$$
\begin{aligned}
(\sigma v)^{\phi \phi^{\dagger}}[g g] & =\frac{7 g_{s}^{4}}{216 \pi m_{\phi}^{2}}-v^{2} \frac{59 g_{s}^{4}}{5184 \pi m_{\phi}^{2}}, \\
(\sigma v)^{\phi \phi^{\dagger}}[f \bar{f}] & =v^{2} \frac{g_{s}^{4}}{432 \pi m_{\phi}^{2}}, \quad \text { for } f \neq u \\
(\sigma v)^{\phi \phi^{\dagger}}[u \bar{u}] & =v^{2}\left[\frac{g_{s}^{4}}{432 \pi m_{\phi}^{2}}-\frac{g_{s}^{2} \lambda_{u}^{2}}{108 \pi\left(m_{\chi}^{2}+m_{\phi}^{2}\right)}+\frac{\lambda_{u}^{4} m_{\phi}^{2}}{48 \pi\left(m_{\chi}^{2}+m_{\phi}^{2}\right)^{2}}\right],
\end{aligned}
$$

for both Dirac and Majorana dark matter. Here, $f$ represents the SM quarks and we have neglected all quark masses in our calculation for a heavy $m_{\phi}$ with $m_{\phi} \gg m_{f}$. For Majorana dark matter, there is an additional annihilation channel with cross section

$$
(\sigma v)^{\phi \phi}[u u]=\frac{\lambda_{u}^{4} m_{\chi}^{2}}{6 \pi\left(m_{\phi}^{2}+m_{\chi}^{2}\right)^{2}}+v^{2} \frac{\lambda_{u}^{4} m_{\chi}^{2}\left(3 m_{\chi}^{4}-18 m_{\chi}^{2} m_{\phi}^{2}-m_{\phi}^{4}\right)}{144 \pi\left(m_{\phi}^{2}+m_{\chi}^{2}\right)^{4}} .
$$

Following refs. [48, 49], we have the effective degrees of freedom as a function of the temperature parameter $x$

$$
g_{\mathrm{eff}}=g_{\chi}+g_{\phi}(1+\Delta)^{3 / 2} e^{-x \Delta},
$$

with $g_{\phi}=6$ (we count $\phi$ and $\phi^{\dagger}$ together) and $g_{\chi}=2(4)$ for Majorana (Dirac) fermion. The effective annihilation cross section for the Dirac case is

$$
(\sigma v)_{\mathrm{eff}}=\frac{1}{2}(\sigma v)^{\chi \bar{\chi}} \frac{g_{\chi}^{2}}{g_{\mathrm{eff}}^{2}}+(\sigma v)^{\chi \phi^{\dagger}} \frac{g_{\chi} g_{\phi}}{g_{\mathrm{eff}}^{2}}(1+\Delta)^{3 / 2} e^{-x \Delta}+\frac{1}{2}(\sigma v)^{\phi \phi^{\dagger}} \frac{g_{\phi}^{2}}{g_{\mathrm{eff}}^{2}}(1+\Delta)^{3} e^{-2 x \Delta},
$$

and for the Majorana case is

$$
(\sigma v)_{\mathrm{eff}}=(\sigma v)^{\chi \chi} \frac{g_{\chi}^{2}}{g_{\mathrm{eff}}^{2}}+(\sigma v)^{\chi \phi^{\dagger}} \frac{g_{\chi} g_{\phi}}{g_{\mathrm{eff}}^{2}}(1+\Delta)^{3 / 2} e^{-x \Delta}+\frac{1}{2}\left[(\sigma v)^{\phi \phi^{\dagger}}+(\sigma v)^{\phi \phi}\right] \frac{g_{\phi}^{2}}{g_{\mathrm{eff}}^{2}}(1+\Delta)^{3} e^{-2 x \Delta},
$$



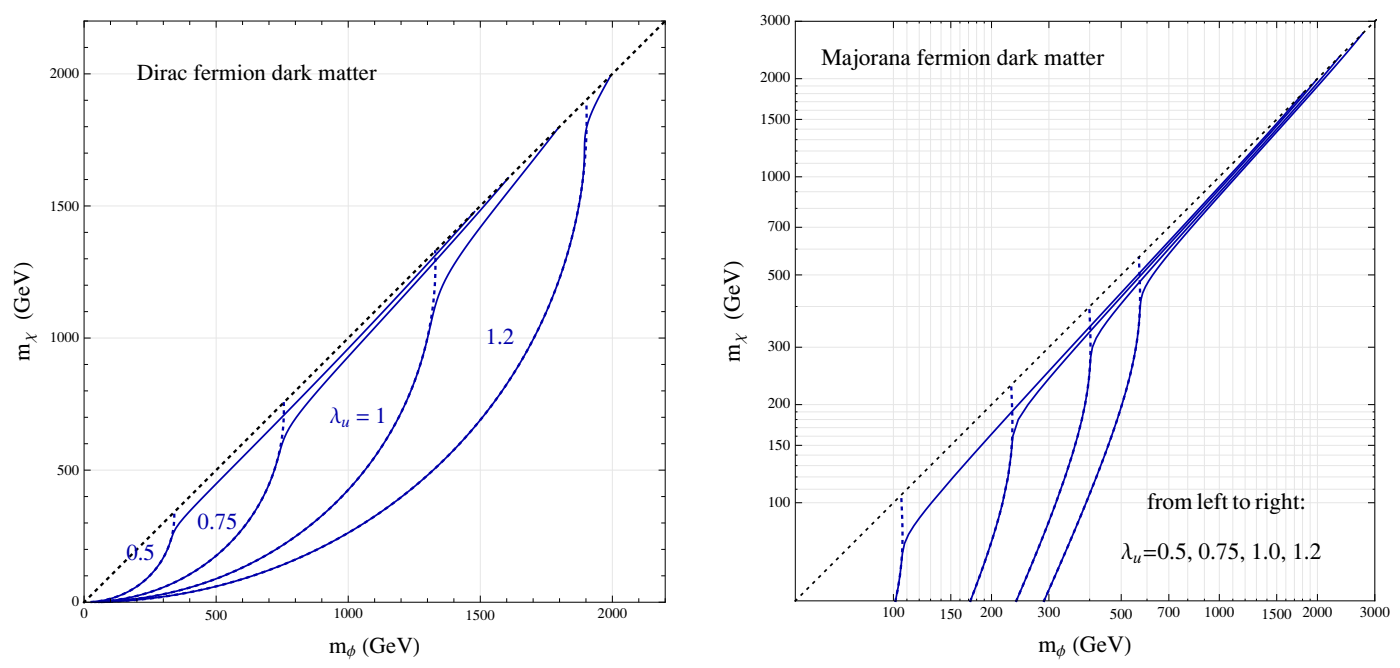

Figure 1. Left panel: the masses of Dirac fermion dark matter and its partner for different choices of coupling, after fitting the observed dark matter energy fraction, $\Omega_{\chi} h^{2}=0.1199 \pm 0.0027$, from Planck [4] and WMAP [3]. The blue dotted lines neglect co-annihilation effects, while the blue solid lines include them. The black dotted line is boundary of the region for which $m_{\phi}>m_{\chi}$. Right panel: the same, but for a Majorana dark matter.

Variables $s_{\text {eff }}$ and $p_{\text {eff }}$ can be constructed by forming a similar combination to $(\sigma v)_{\text {eff }}$. They replace $s$ and $p$ in eqs. (3.3) and (3.4) for the purposes of calculating the thermal relic abundance.

Fitting to the observed value of $\Omega_{\chi} h^{2}=0.1199 \pm 0.0027$ from Planck [4] and WMAP [3], we show the allowed values of $m_{\chi}$ and $m_{\phi}$ in figure 1 for different values of couplings. For Dirac dark matter, the co-annihilation effects have a significant effect for small values of $\lambda_{u}$, but only have a small effect for lager values of $\lambda_{u}$. Due to $p$-wave suppression of $\chi \chi$ annihilation, Majorana dark matter mass is preferred to have either a light mass, below around $600 \mathrm{GeV}$, or a heavy mass nearly degenerate with its partner.

For complex scalar dark matter, the annihilation rate of $X X^{\dagger} \rightarrow u \bar{u}$ is also $p$-wave suppressed and given by

$$
\frac{1}{2}(\sigma v)_{\text {complex scalar }}^{X X^{\dagger}}=\frac{1}{2}\left[v^{2} \frac{\lambda^{4} m_{X}^{2}}{16 \pi\left(m_{X}^{2}+m_{\psi}^{2}\right)^{2}}\right] \equiv p v^{2} .
$$

The allowed parameter space for a thermal relic in the complex scalar case has similar features to the Majorana case, including the co-annihilation effects.

\section{Dark matter direct detection}

For calculation of dark matter direct detection cross-sections, one could integrate out the dark matter partner and calculate the scattering cross sections using the effective operators. However, for the degenerate region, the dark matter partner in the $s$-channel can dramatically increase the scattering cross section. To capture the resonance effects, we keep the dark matter partner propagator in our calculation. 


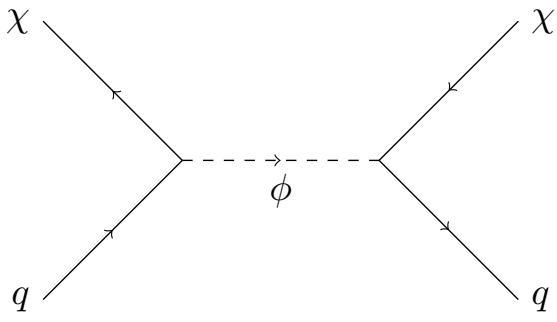

(a)

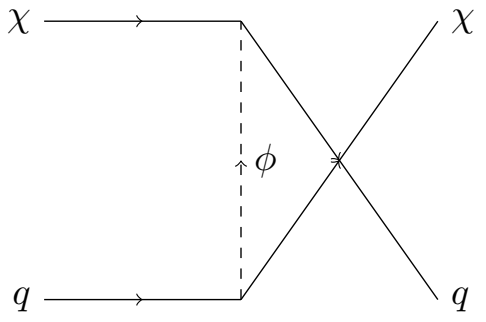

(b)

Figure 2. Feynman diagrams for scattering of a fermion dark matter off nucleus. Only the left panel in (a) contributes to the Dirac fermion case, while both (a) and (b) contribute to the Majorana fermion case.

For the Dirac dark matter case, only the left panel in figure 2 contributes. Both spinindependent (SI) and spin-dependent (SD) scattering exist. The leading SI interaction cross-section per nucleon is given by

$$
\sigma_{\mathrm{SI}}^{N q}(\operatorname{Dirac})=\frac{\left|\lambda_{u}\right|^{4} f_{N q}^{2} \mu^{2}}{64 \pi\left[\left(m_{\chi}^{2}-m_{\phi}^{2}\right)^{2}+\Gamma_{\phi}^{2} m_{\phi}^{2}\right]},
$$

where $N=p, n ; \mu$ is the reduced mass of the dark matter-nucleon system; $f_{N q}$ is the coefficient related to the quark operator matrix element inside a nucleon. For the up quark operator at hand, one has $f_{p u}=2$ and $f_{n u}=1[44,50]$. The sub-leading SD interaction cross section is given by

$$
\sigma_{\mathrm{SD}}^{N q}(\text { Dirac, Majorana })=\frac{3\left|\lambda_{u}\right|^{4} \Delta_{N q}^{2} \mu^{2}}{64 \pi\left[\left(m_{\chi}^{2}-m_{\phi}^{2}\right)^{2}+\Gamma_{\phi}^{2} m_{\phi}^{2}\right]},
$$

with $\Delta_{u}^{p}=\Delta_{d}^{n}=0.842 \pm 0.012$ and $\Delta_{d}^{p}=\Delta_{u}^{n}=-0.427 \pm 0.013$ [51]. For Majorana dark matter, there is only an SD scattering cross section with the same formula as the SD scattering of the Dirac fermion case.

For the complex scalar case, the SI scattering cross section is given by

$$
\sigma_{\mathrm{SI}}^{N q}(\text { complex scalar })=\frac{\left|\lambda_{u}\right|^{4} f_{N q}^{2} m_{p}^{2}}{32 \pi\left[\left(m_{X}^{2}-m_{\psi}^{2}\right)^{2}+\Gamma_{\psi}^{2} m_{\psi}^{2}\right]},
$$

while the SD scattering cross section is suppressed by the dark matter velocity and is neglected here.

Searches for SI dark matter interactions with nuclei are particularly constraining when they are predicted by a given model. We include the most stringent SI direct detection constraints from Xenon100 [52] for heavier dark matter masses and Xenon10 [53] for lighter dark matter masses. Xenon100 [52] is sensitive to cross-sections nearly down to $10^{-45} \mathrm{~cm}^{2}$ at a dark matter mass of around $100 \mathrm{GeV}$. The Xenon10 [53] experiment has some additional sensitivity for low dark matter masses. For the SD scattering cross section, we mainly use the limits from SIMPLE [54], COUPP [55], and PICASSO [56] experiments for coupling to protons and from Xenon100 [57] and CDMS [58, 59] for coupling to neutrons. 


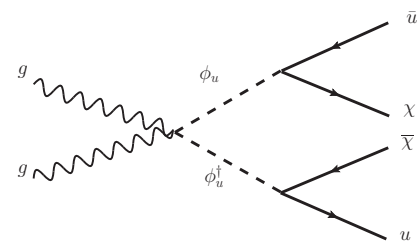

(a)

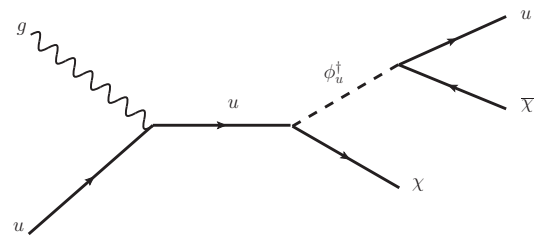

(b)

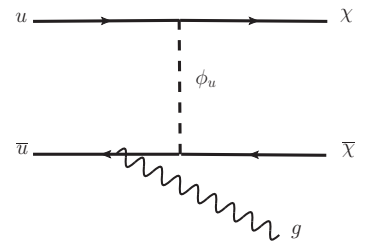

(c)

Figure 3. The three dark matter particle production mechanisms at hadron colliders. Diagram (a) has two jets in final state, while (b) and (c) provide mono-jet signatures.

In addition to placing strong constraints, four experiments (DAMA [60], CoGeNT [61], CRESST-II [62], CDMS [63]) have now seen excesses in regions of parameter space already probed by Xenon100 under some assumptions [64]. For the purposes of this study, we ignore the debatable excesses and only consider the constraints from experiments. Because we study the up quark and down quark operators separately, isospin symmetry is generically broken. Therefore, we will consider the constraints on dark matter-proton and dark matter-neutron scattering separately for both SI and SD.

\section{Collider constraints}

Since the dark matter couples to quarks, it can be produced at colliders. In addition, the colored mediator may be produced, yielding strong constraints both from associated and pair production. Except in the regime of an extremely heavy mediator, these channels provide the dominant constraints. Associated production of the mediator and the dark matter particle, along with radiative contributions from dark matter pair production, yield a monojet signature, while pair production of mediators can be seen in searches for jets plus missing transverse energy (MET). Example diagrams for the three production mechanisms in the case of coupling to up quarks are illustrated in figure 3. For simplicity, we consider the constraints from the CMS experiment in the monojet and jets plus MET channels $[25,65]$. There are comparable constraints from the ATLAS experiment $[21,66]$, though the reach of the CMS searches is slightly better at present.

\subsection{Estimated limits from monojet on $t$-change $\phi$ exchange}

For the fermionic dark matter case and in the heavy $m_{\phi}$ limit, the Fierz-transformed effective operator

$$
\frac{\left|\lambda_{u}\right|^{2}}{8 m_{\phi}^{2}} \bar{\chi} \gamma_{\mu}\left(1+\gamma_{5}\right) \chi \bar{u} \gamma^{\mu}\left(1-\gamma_{5}\right) u
$$

is generated. The existing search at the $8 \mathrm{TeV}$ LHC with around $20 \mathrm{fb}^{-1}$ constrains the combination of up quark and down quark operators. For light dark matter masses below analysis cuts on monojet $p_{T}$ or $\mathbb{E}_{T}$, the collider production cross section is insensitive to the parity structure of the operators [25]. One can approximately translate the constraints on $\Lambda \sim \sqrt{2} m_{\phi} /\left|\lambda_{u}\right|$ obtained in ref. [25] to our model parameter space. For light dark matter 


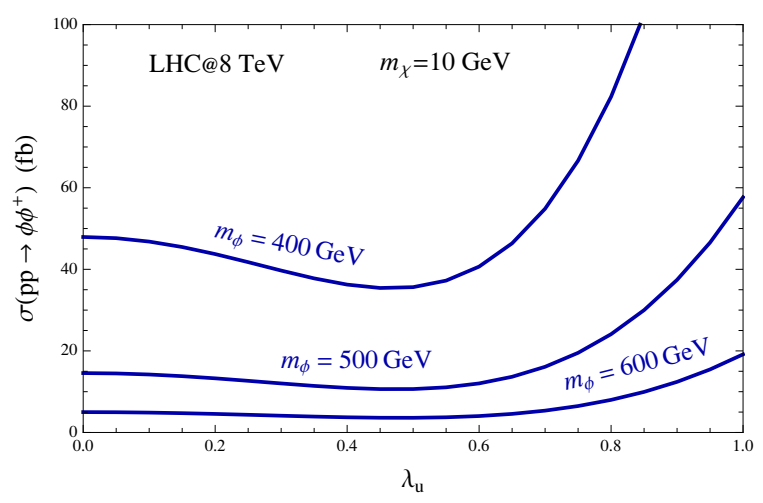

Figure 4. The pair-production cross sections of the $\phi$ field as a function of $\lambda_{u}$.

masses, the $90 \%$ confidence level (CL) constraints on $\Lambda$ in ref. [25] is around $900 \mathrm{GeV}$, leading to an estimated constraint of $m_{\phi} /\left|\lambda_{u}\right| \gtrsim 640 \mathrm{GeV}$.

\subsection{Limits from $2 j+E_{T}^{\text {miss }}$ on $\phi$ pair production}

In the limit of a small dark matter-mediator coupling, $\lambda_{u} \approx 0$, the only significant diagram yielding this final state is (a) in figure 3 . The production cross-section is identical to that of a single squark in the MSSM. The present bounds on this process from CMS constrain the colored particle mass to be above around $500 \mathrm{GeV}$ [67] for a massless neutralino. For $\lambda_{u} \neq 0$, there are additional contributions from $t$-channel dark matter exchange and the cross-section for the parton level process $u+\bar{u} \rightarrow \phi+\phi^{*}$ is given by:

$$
\begin{aligned}
& \sigma=-\frac{1}{1728 \pi s^{3}}\left\{2 \sqrt{s\left(s-4 m_{\phi}^{2}\right)}\left[4 g_{s}^{4}\left(4 m_{\phi}^{2}-s\right)+12 g_{s}^{2} \lambda_{u}^{2}\left(s+2 m_{\chi}^{2}-2 m_{\phi}^{2}\right)+27 \lambda_{u}^{4} s\right]\right. \\
& \left.+3 \lambda_{u}^{2}\left[16 g_{s}^{2}\left(m_{\chi}^{2} s+\left(m_{\phi}^{2}-m_{\chi}^{2}\right)^{2}\right)+9 \lambda_{u}^{2} s\left(s+2 m_{\chi}^{2}-2 m_{\phi}^{2}\right)\right] \log \left[\frac{s-\sqrt{s\left(s-4 m_{\phi}^{2}\right)}+2 m_{\chi}^{2}-2 m_{\phi}^{2}}{s+\sqrt{s\left(s-4 m_{\phi}^{2}\right)}+2 m_{\chi}^{2}-2 m_{\phi}^{2}}\right]\right\} .
\end{aligned}
$$

This extra contribution is significant for $\lambda_{u}=1$ and leads to a much higher sensitivity. We also note that there is destructive interference for a small value of $\lambda_{u}$, as shown in figure 4 for different values of $m_{\phi}$. We therefore anticipate that the experimental limits from jets plus $E_{T}^{\text {miss }}$ could become weaker at some intermediate values of $\lambda_{u}$.

To estimate the current bounds on this model, as well as the case of scalar dark matter, we calculate LO cross-sections for the full process using MadGraph [68] with a model constructed by FeynRules [69]. NLO K-factors calculated using Prospino [70] are applied to the pure QCD contribution to the cross-section for the cases of fermionic dark matter. The limits provided in [65] are then applied to the calculated cross-section to obtain an estimate of the current 95\% CL exclusion limit. The results of this analysis are presented below, in section 5.3 . 

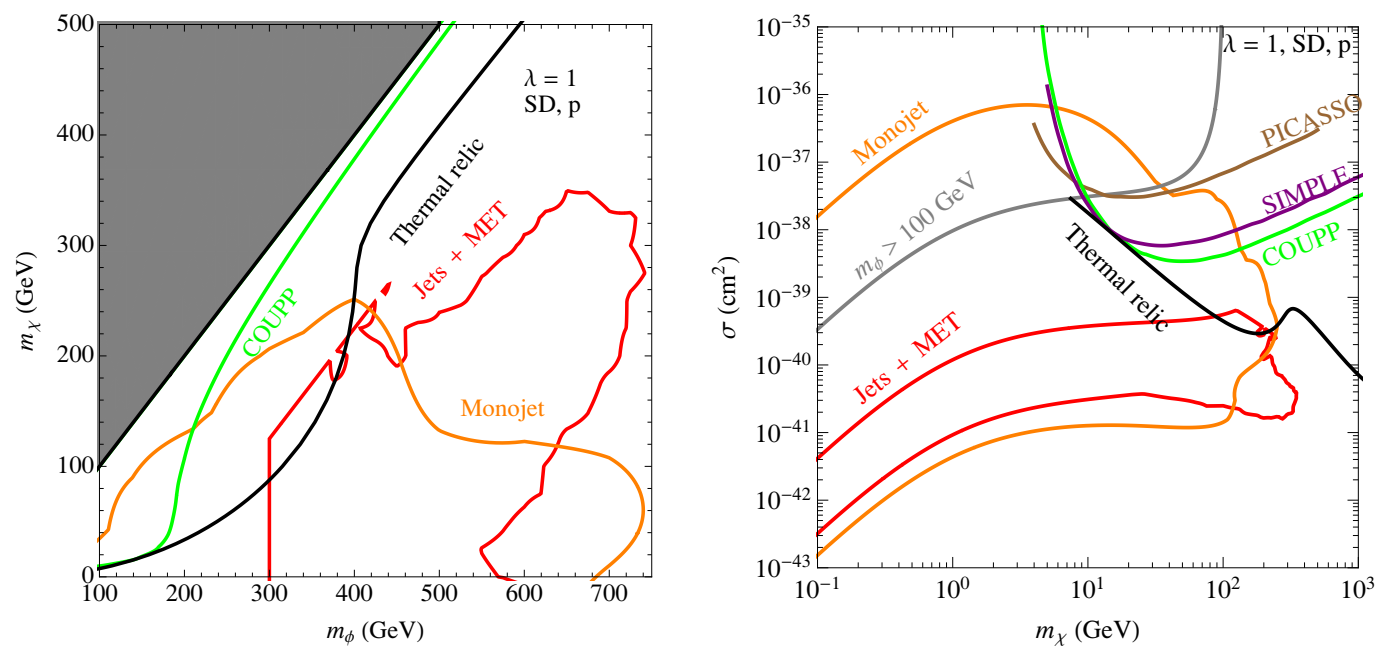

Figure 5. 95\% exclusion limits (except the black solid line from the thermal relic abundance) from the most sensitive searches for Majorana dark matter with the only coupling to the up quark with $\lambda_{u}=1$. The left panel is in the $m_{\phi}-m_{\chi}$ plane, while the right panel is in the $\sigma-m_{\chi}$ plane.

\subsection{Limits from monojet on single $\phi$ productions}

The dominant production channel for monojets is process (b) in figure 3 at a small value of $\lambda_{u}$. The resulting cross-section at LO for $u+g \rightarrow \phi+\chi$ is given by

$$
\sigma(u+g \rightarrow \phi+\chi)=\frac{\lambda_{u}^{2} g_{s}^{2}}{768 \pi s^{3}}\left(3 s+2 m_{\chi}^{2}-2 m_{\phi}^{2}\right) \sqrt{\left(s+m_{\chi}^{2}-m_{\phi}^{2}\right)^{2}-4 m_{\chi}^{2} s},
$$

where $\sqrt{s}$ is the center-of-mass energy. In order to estimate the current reach of monojet searches, we generate events for all tree-level diagrams with one quark plus dark matter particles in the final state using MadGraph [68] with the models defined in FeynRules [69]. The events are showered and hadronized using Pythia [71], then the hadrons are clustered into jets using FastJet [72]. The cuts described in ref. [25] are then applied to the events in order to estimate the acceptance times efficiency of that search. The resulting LO signal cross section times estimated efficiency and acceptance for each signal region are compared to the limits set in ref. [25]. We present our results for several different scenarios in two ways: first in the $m_{\phi}-m_{\chi}$ plane and second in the $m_{\chi}-\sigma_{\mathrm{SI}(\mathrm{SD})}$ plane with all limits at $95 \% \mathrm{CL}$.

We begin by considering the model with Majorana dark matter and only $\lambda_{u} \neq 0$. For $\lambda_{u}=1$, the exclusion curves are shown in figure 5 . The dominant constraints come from collider searches in the monojet and jets + MET channels, as well as dark matter spin-dependent direct detection searches. In addition, we show the lines at which the observed dark matter relic abundance is attained assuming that $\chi$ is a thermal relic. The exclusion extends up to scalar masses of around $700 \mathrm{GeV}$ provided that the dark matter is lighter than about $300 \mathrm{GeV}$. In figure 5, we have included the co-annihilation effects for the degenerate spectrum. We show the thermal relic required parameter space in the black and solid line in both panels of figure 5 . In the $\sigma-m_{\chi}$ plane, we stop plotting the thermal relic line when the dark matter mass is close to the mediator mass. There is some 

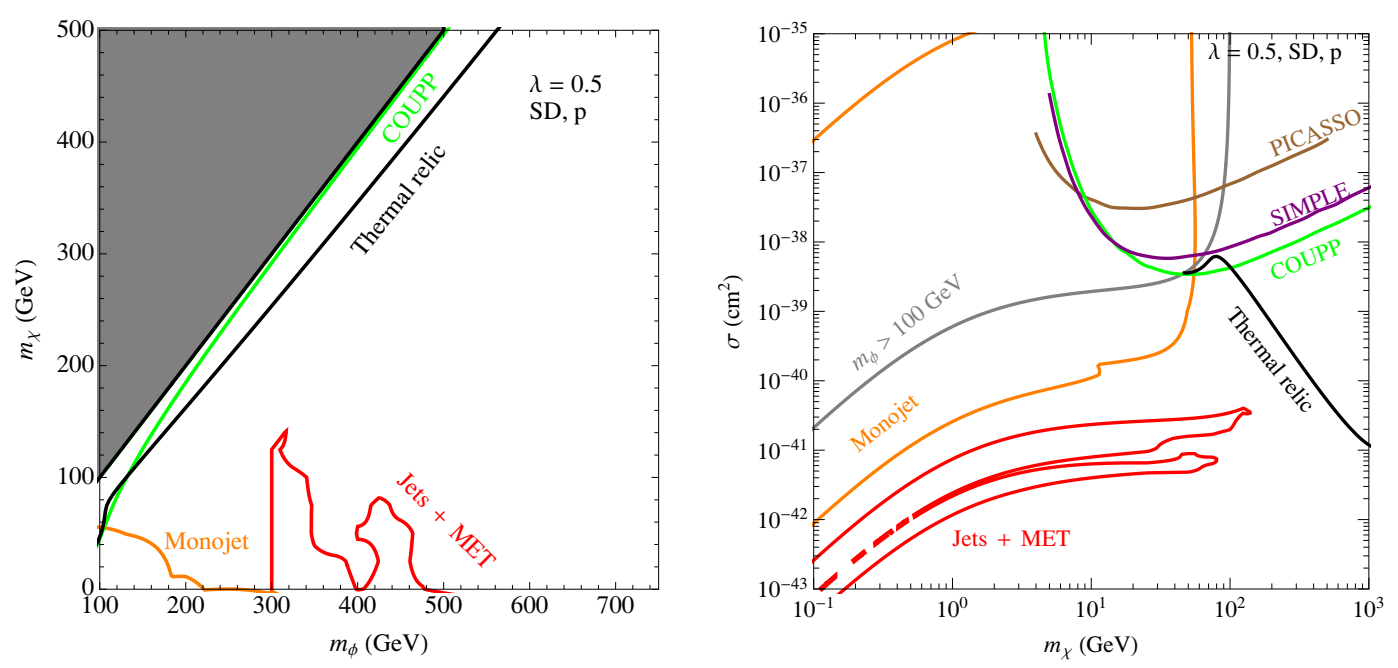

Figure 6. The same as figure 5 for the up quark case with $\lambda_{u}=0.5$.

parameter space at the moment where a thermal relic is allowed, for a mediator mass of around $400 \mathrm{GeV}$, though we stress that the thermal relic abundance may be set in other ways. It is important to note that in this model, the monojet search has a wider reach than the jets + MET search for heavy mediator masses. This is due to the fact that some of the diagrams for $\phi \phi$ production are proportional to the Majorana dark matter mass. In addition, up to dark matter masses of around $300 \mathrm{GeV}$, the dominant constraint on these models comes from colliders. In particular, this means that the possibility of light dark matter below a few $\mathrm{GeV}$ is highly constrained. The SD direct detection, jets+MET and monojet are complimentary as they cover different parts of parameter space.

For comparison, in figure 6 we show the same exclusions in the mass plane for $\lambda_{u}=0.5$. In this case, the current constraints are far weaker. Even for the mediator masses below a few hundred $\mathrm{GeV}$, there is a significant allowed fraction of parameter space, which it is important to cover in future searches, especially at colliders. On the other hand, for such a small coupling, it is difficult to obtain the correct relic abundance via thermal production except in the co-annihilation region; an alternate non-thermal mechanism could be considered such that dark matter is not over-produced.

We also study the same model, but for the down quark case with only $\lambda_{d} \neq 0$. For $\lambda_{d}=1$, the exclusion curves are shown in figures 7 . The dominant constraints are the same as in the up-type case. The constraints are slightly weaker in this case and the jets + MET search dominates for at high mediator masses as it is less sensitive to the down quark parton distribution function suppression. In this case, there is a similar parameter space allowed for a thermal relic.

Next, we consider models with Dirac dark matter and complex scalar dark matter. For these models, the SI direct detection constraints dominate up to very low dark matter masses, independent of $m_{\phi}$. For $\lambda_{u}=1$, the exclusion curves are shown in figures 8 and 9 . These cases are highly constrained by searches for spin-independent scattering, which is unsuppressed. Since dark matter interactions generally violate isospin in our models, the 

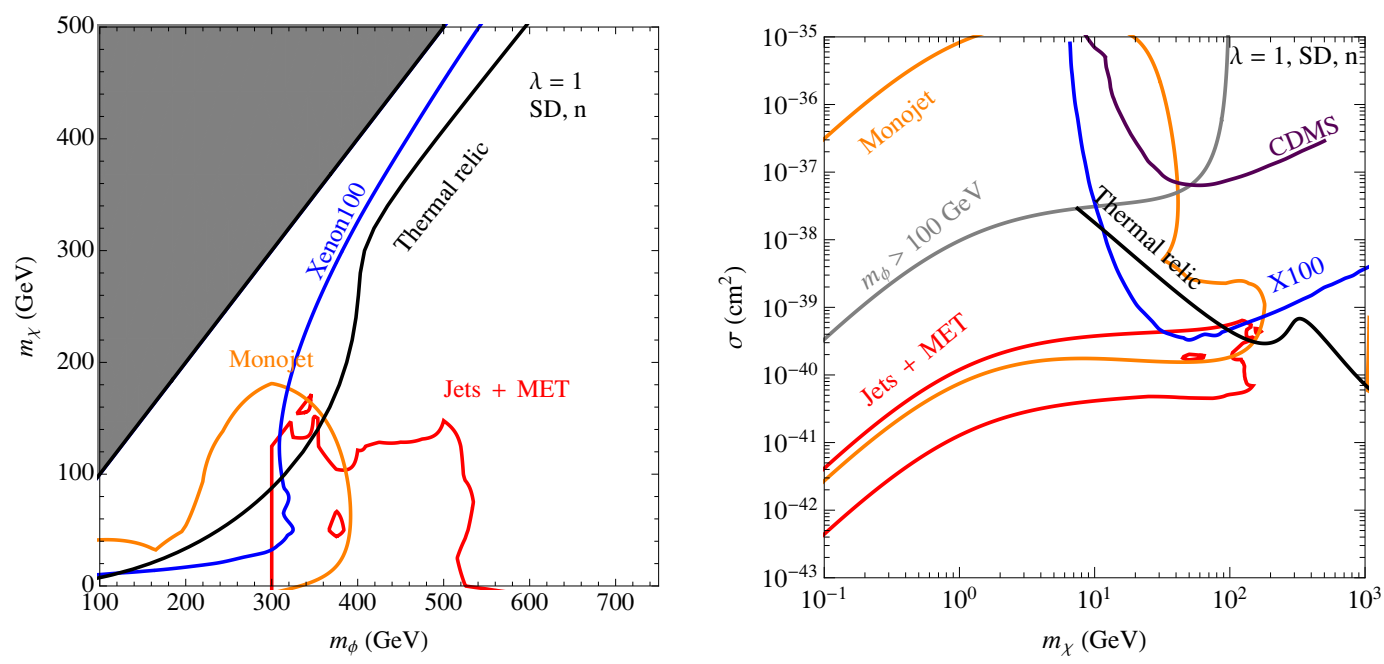

Figure 7. $95 \%$ exclusion limits from the most sensitive searches for Majorana dark matter with coupling to the down quark.
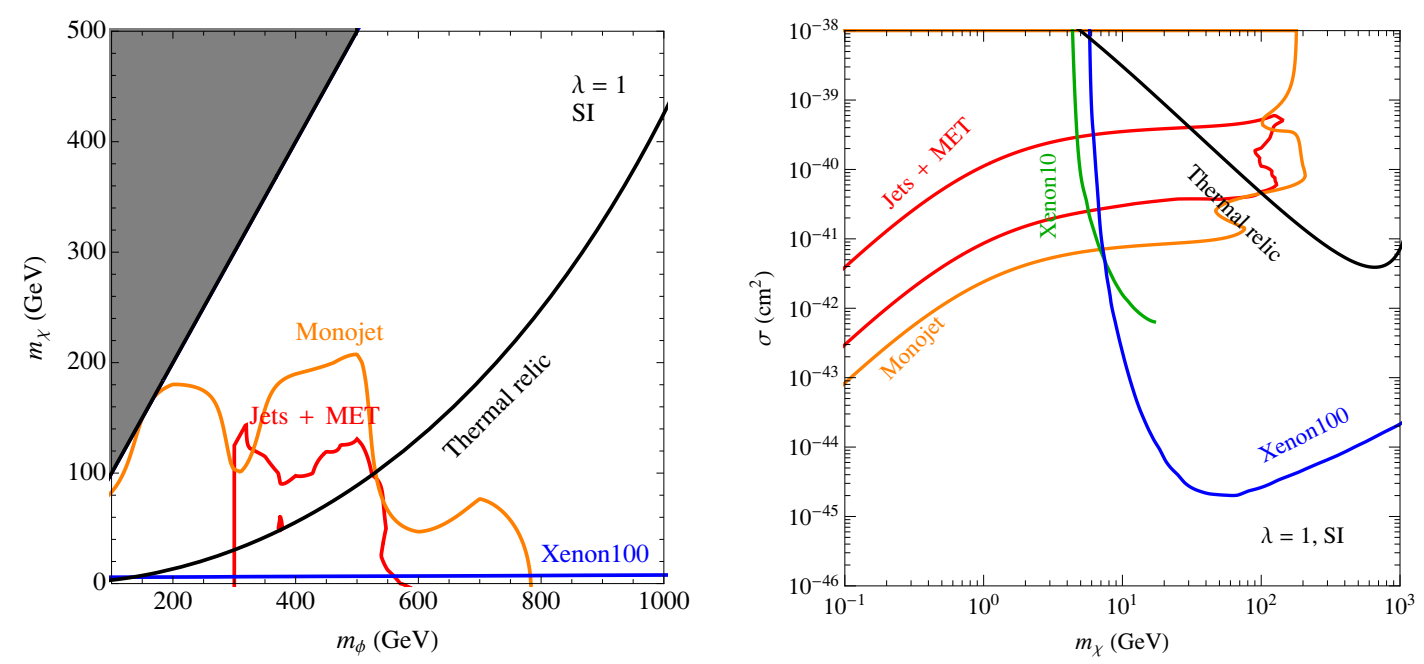

Figure 8. 95\% exclusion limits from the most sensitive searches for Dirac dark matter with coupling to the up quark.

different couplings to protons and neutrons should be taken into account in calculating the bounds. The SI cross-section bounds per nucleon are generally calculated under the assumption of isospin, such that the proton and neutron cross-sections are the same. In order to take into account isospin violation, we calculate the cross-section for interaction with a proton and rescale by

$$
\sigma_{\mathrm{DM}, \text { nucleon }}=\frac{\left[f_{p} Z+f_{n}(A-Z)\right]^{2}}{f_{p}^{2} A^{2}} \sigma_{\mathrm{DM}, p},
$$

where $A$ and $Z$ are the mass number and atomic number of the target nucleus respectively. The dominant SI bounds come from Xe targets, so that $A=131$, neglecting small effects 

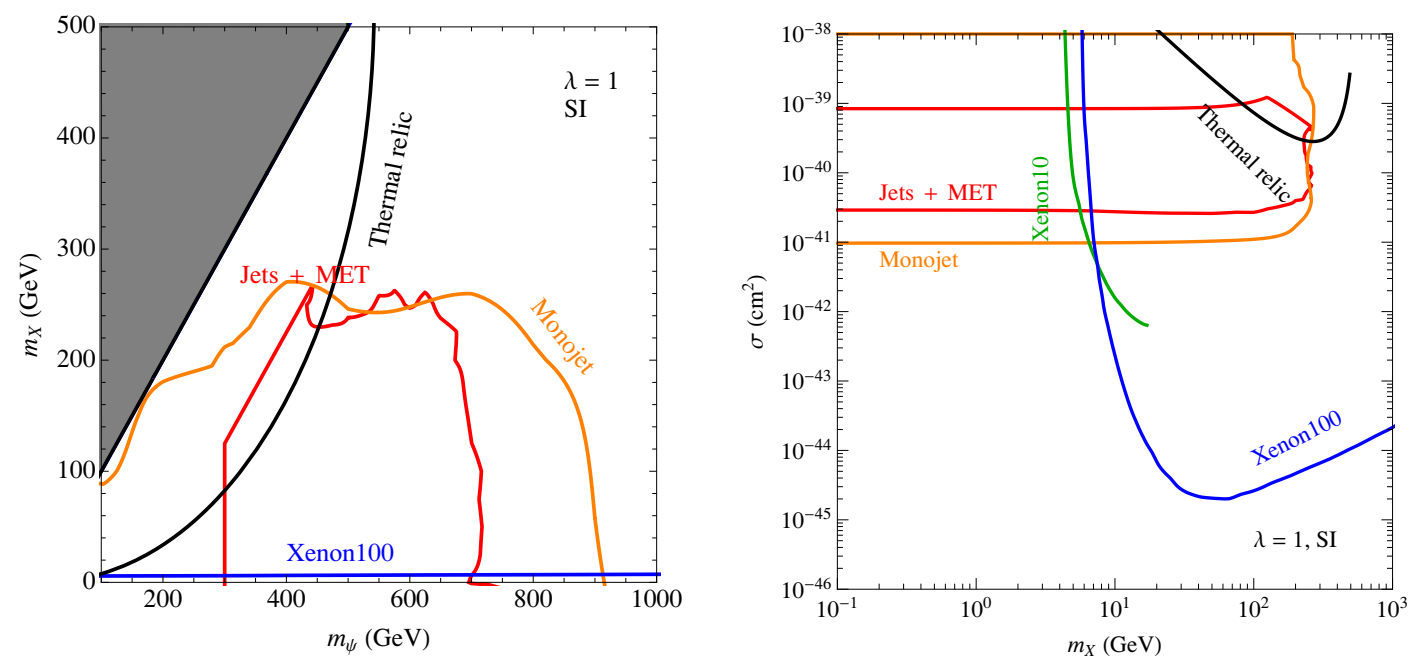

Figure 9. 95\% exclusion limits from the most sensitive searches for complex scalar dark matter with coupling to the up quark.

from other comparable or subdominant isotopes, and $Z=54$. All scattering cross sections presented in figures 8 and 9 are the averaged one, $\sigma_{\mathrm{DM}, \text { nucleon}}$.

It is interesting to note that collider bounds take over for light dark matter, below the threshold of direct detection experiments. In the case of a complex scalar, the low mass bound flattens out in the cross-section plane since it is not sensitive to the reduced mass of the dark matter-nucleon system, but rather the nucleon mass itself, as can be seen from eq. (4.3).

\section{Discussion and conclusions}

The signal spectrum from the associated production of dark matter and its partner could be dramatically different from backgrounds. Particularly when the Yukawa coupling is small, associated production is the dominant part of the signal. Additional kinematic variables can be used to enhance the dark matter signal in the fermion-portal scenario. We use MadGraph5 [68] to generate the dark matter signal events and shower them in PYTHIA [73]. We then use PGS [74] to perform the fast detector simulation. After utilizing the basic cuts in ref. [25], where $E_{T}^{\text {miss }}>200 \mathrm{GeV}$ has been imposed, we calculate the normalized $E_{T}^{\text {miss }}$ distributions for several different spectra. In the left panel of figure 10, we show the $E_{T}^{\text {miss }}$ from the $\chi+\phi$ associate productions. Because the jet from the decay of $\phi \rightarrow \chi+j$ is energetic, the $E_{T}^{\text {miss }}$ distributions have a peak-structure with the peak at around $m_{\phi} / 2$ for a small $m_{\chi}$. As a comparison, the right panel of the figure 10 shows the $E_{T}^{\text {miss }}$ distribution without onshell production of $\phi$. The spectrum is monotonically decreasing in this case, which follows the shape of the background although with a different slope. For a larger $m_{\phi}$, the signal spectrum becomes slightly harder at higher masses. In principle, the peak structure in the left panel can be used to discover dark matter, for instance performing a "bump" search in 

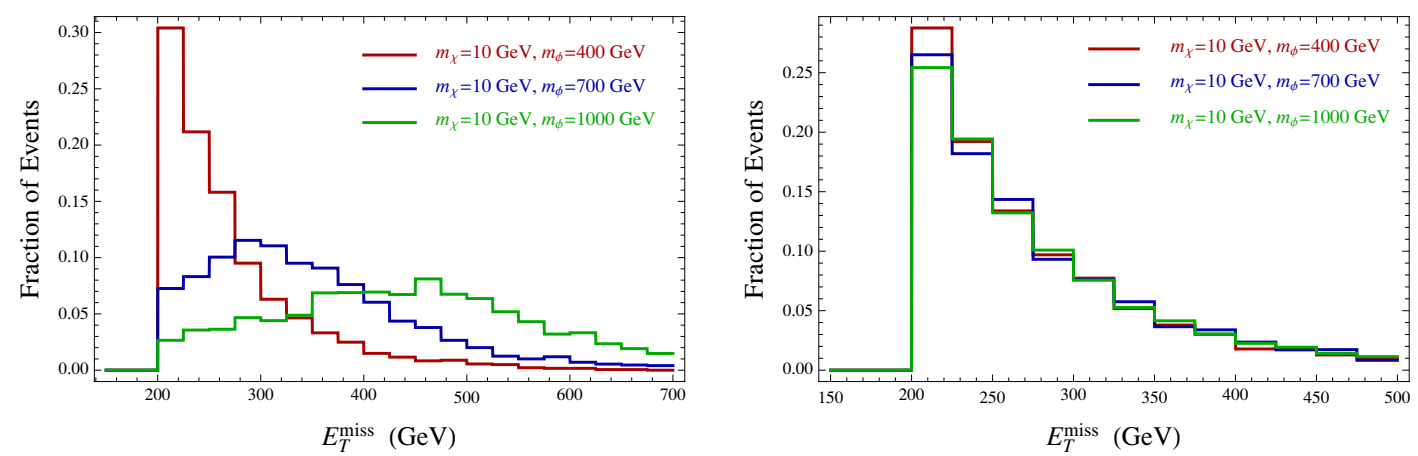

Figure 10. Left panel: the fraction of events after basic cuts as a function of $E_{T}^{\text {miss }}$ for the associated production of $\chi+\phi$ with $\phi \rightarrow \chi+j$. Right panel: the same as the left one but for the productions of $2 \chi+j$ with the jet from ISR.

the $E_{T}^{\text {miss }}$ distribution. In practice, the peaks are too wide to make it feasible. Improving the jet energy resolution and $E_{T}^{\text {miss }}$ measurement can yield significant boosts in sensitivity.

To explore more fermion portal dark matter parameter space, we emphasize the importance of a dedicated search of the two jets plus MET signature. As can be seen from the left panel in figure 6, for small values of the Yukawa coupling, the current limit on the colored mediator mass is weak, around $350 \mathrm{GeV}$ for a dark matter mass at $100 \mathrm{GeV}$. Additional kinematic variables like $m_{T_{2}}$ can increase the search sensitivity [75].

In this paper, we have concentrated on the complimentarity of collider and direct detection searches. The indirect searches for dark matter should also play a role, although the current constraint is not that sensitive. For instance, we have checked that for $\lambda=1$, a dark matter mass of $100 \mathrm{GeV}$ and any mediator mass above the dark matter mass, the new contribution to the anti-proton flux from Dirac fermion dark matter annihilations is at least one order of magnitude below the measured flux at PAMELA [76]. Because of the $p$-wave suppression for the Majorana fermion and complex scalar dark matter annihilation cases, the indirect detection constraints are even weaker.

Note added: we note here that during the completion of our paper, another paper [77] appeared with different emphasis: our paper concentrates more on the complimentarity of direct detection and collider searches for dark matter, while their paper has more focus on the thermal relic parameter space.

\section{Acknowledgments}

Y. Bai is supported by startup funds from the UW-Madison. SLAC is operated by Stanford University for the US Department of Energy under contract DE-AC02-76SF00515. YB also would like to thank the Kvali Institute for Theoretical Physics, U. C. Santa Barbara, where part of this work was done. This research was also supported in part by the National Science Foundation under Grant No. NSF PHY11-25915. 
Open Access. This article is distributed under the terms of the Creative Commons Attribution License which permits any use, distribution and reproduction in any medium, provided the original author(s) and source are credited.

\section{References}

[1] K. Begeman, A. Broeils and R. Sanders, Extended rotation curves of spiral galaxies: dark haloes and modified dynamics, Mon. Not. Roy. Astron. Soc. 249 (1991) 523 [inSPIRE].

[2] M. Bradac et al., Strong and weak lensing united. 3. Measuring the mass distribution of the merging galaxy cluster 1E0657-56, Astrophys. J. 652 (2006) 937 [astro-ph/0608408] [INSPIRE].

[3] WMAP collaboration, C. Bennett et al., Nine-year Wilkinson Microwave Anisotropy Probe (WMAP) observations: final maps and results, Astrophys. J. Suppl. 208 (2013) 20 [arXiv: 1212.5225] [INSPIRE].

[4] Planck collaboration, P. Ade et al., Planck 2013 results. XVI. Cosmological parameters, arXiv: 1303.5076 [INSPIRE].

[5] A. Birkedal, K. Matchev and M. Perelstein, Dark matter at colliders: a model independent approach, Phys. Rev. D 70 (2004) 077701 [hep-ph/0403004] [INSPIRE].

[6] V. Barger, W.-Y. Keung and G. Shaughnessy, Spin dependence of dark matter scattering, Phys. Rev. D 78 (2008) 056007 [arXiv:0806. 1962] [inSPIRE].

[7] Q.-H. Cao, C.-R. Chen, C.S. Li and H. Zhang, Effective dark matter model: relic density, CDMS II, Fermi LAT and LHC, JHEP 08 (2011) 018 [arXiv:0912.4511] [INSPIRE].

[8] M. Beltrán, D. Hooper, E.W. Kolb, Z.A. Krusberg and T.M. Tait, Maverick dark matter at colliders, JHEP 09 (2010) 037 [arXiv: 1002.4137] [INSPIRE].

[9] Y. Bai, P.J. Fox and R. Harnik, The Tevatron at the frontier of dark matter direct detection, JHEP 12 (2010) 048 [arXiv: 1005.3797] [INSPIRE].

[10] J. Goodman et al., Constraints on light Majorana dark matter from colliders, Phys. Lett. B 695 (2011) 185 [arXiv:1005.1286] [InSPIRE].

[11] J. Goodman et al., Constraints on dark matter from colliders, Phys. Rev. D 82 (2010) 116010 [arXiv: 1008.1783] [InSPIRE].

[12] J. Fan, M. Reece and L.-T. Wang, Non-relativistic effective theory of dark matter direct detection, JCAP 11 (2010) 042 [arXiv:1008.1591] [InSPIRE].

[13] M.R. Buckley, Asymmetric dark matter and effective operators, Phys. Rev. D 84 (2011) 043510 [arXiv:1104.1429] [INSPIRE].

[14] A. Rajaraman, W. Shepherd, T.M. Tait and A.M. Wijangco, LHC bounds on interactions of dark matter, Phys. Rev. D 84 (2011) 095013 [arXiv:1108.1196] [INSPIRE].

[15] P.J. Fox, R. Harnik, J. Kopp and Y. Tsai, Missing energy signatures of dark matter at the LHC, Phys. Rev. D 85 (2012) 056011 [arXiv:1109.4398] [inSPIRE].

[16] K. Cheung, P.-Y. Tseng, Y.-L.S. Tsai and T.-C. Yuan, Global constraints on effective dark matter interactions: relic density, direct detection, indirect detection and collider, JCAP 05 (2012) 001 [arXiv: 1201.3402] [INSPIRE]. 
[17] CDF collaboration, T. Aaltonen et al., A search for dark matter in events with one jet and missing transverse energy in $\bar{p}$ collisions at $\sqrt{s}=1.96 \mathrm{Te} V$,

Phys. Rev. Lett. 108 (2012) 211804 [arXiv:1203.0742] [INSPIRE].

[18] A.L. Fitzpatrick, W. Haxton, E. Katz, N. Lubbers and Y. Xu, The effective field theory of dark matter direct detection, JCAP 02 (2013) 004 [arXiv:1203.3542] [INSPIRE].

[19] V. Barger, W.-Y. Keung, D. Marfatia and P.-Y. Tseng, Dipole moment dark matter at the LHC, Phys. Lett. B 717 (2012) 219 [arXiv:1206.0640] [InSPIRE].

[20] Y. Bai and T.M. Tait, Searches with mono-leptons, Phys. Lett. B 723 (2013) 384 [arXiv:1208.4361] [INSPIRE].

[21] ATLAS collaboration, Search for new phenomena in monojet plus missing transverse momentum final states using $10 \mathrm{fb}^{-1}$ of pp collisions at $\sqrt{s}=8$ TeV with the ATLAS detector at the LHC, ATLAS-CONF-2012-147, CERN, Geneva Switzerland (2012).

[22] Y.J. Chae and M. Perelstein, Dark matter search at a linear collider: effective operator approach, JHEP 05 (2013) 138 [arXiv:1211.4008] [INSPIRE].

[23] H. Dreiner, D. Schmeier and J. Tattersall, Contact interactions probe effective dark matter models at the LHC, Europhys. Lett. 102 (2013) 51001 [arXiv:1303.3348] [INSPIRE].

[24] J.M. Cornell, S. Profumo and W. Shepherd, Kinetic decoupling and small-scale structure in effective theories of dark matter, Phys. Rev. D 88 (2013) 015027 [arXiv:1305.4676] [INSPIRE].

[25] CMS collaboration, Search for new physics in monojet events in pp collisions at $\sqrt{s}=8 \mathrm{TeV}$, CMS-PAS-EXO-12-048, CERN, Geneva Switzerland (2012).

[26] I.M. Shoemaker and L. Vecchi, Unitarity and monojet bounds on models for DAMA, CoGeNT and CRESST-II, Phys. Rev. D 86 (2012) 015023 [arXiv:1112.5457] [InSPIRE].

[27] H. An, R. Huo and L.-T. Wang, Searching for low mass dark portal at the LHC, Phys. Dark Univ. 2 (2013) 50 [arXiv:1212.2221] [INSPIRE].

[28] G. Busoni, A. De Simone, E. Morgante and A. Riotto, On the validity of the effective field theory for dark matter searches at the LHC, arXiv: 1307.2253 [INSPIRE].

[29] ATLAS collaboration, Search for dark matter pair production in events with a hadronically decaying $W$ or $Z$ boson and missing transverse momentum in pp collision data at $\sqrt{s}=8$ TeV with the ATLAS detector, ATLAS-CONF-2013-073, CERN, Geneva Switzerland (2013).

[30] CMS collaboration, Search for dark matter in the mono-lepton channel with pp collision events at center-of-mass energy of $8 \mathrm{TeV}$, CMS-PAS-EXO-13-004, CERN, Geneva Switzerland (2013).

[31] M. Cahill-Rowley et al., Complementarity and searches for dark matter in the pMSSM, arXiv:1305.6921 [INSPIRE].

[32] R.E. Shrock and M. Suzuki, Invisible decays of Higgs bosons, Phys. Lett. B 110 (1982) 250 [INSPIRE].

[33] C. Burgess, M. Pospelov and T. ter Veldhuis, The minimal model of nonbaryonic dark matter: a singlet scalar, Nucl. Phys. B 619 (2001) 709 [hep-ph/0011335] [INSPIRE].

[34] B. Patt and F. Wilczek, Higgs-field portal into hidden sectors, hep-ph/0605188 [INSPIRE]. 
[35] Y. Bai, V. Barger, L.L. Everett and G. Shaughnessy, 2HDM portal dark matter: LHC data and the Fermi-LAT 135 GeV line, Phys. Rev. D 88 (2013) 015008 [arXiv:1212.5604] [INSPIRE].

[36] Y. Nomura and J. Thaler, Dark matter through the axion portal, Phys. Rev. D 79 (2009) 075008 [arXiv:0810.5397] [inSPIRE].

[37] H.M. Lee, M. Park and V. Sanz, Gravity-mediated dark matter, arXiv:1306.4107 [INSPIRE].

[38] Y. Bai, M. Carena and J. Lykken, Dilaton-assisted dark matter, Phys. Rev. Lett. 103 (2009) 261803 [arXiv:0909.1319] [INSPIRE].

[39] K. Cheung and T.-C. Yuan, Hidden fermion as milli-charged dark matter in Stueckelberg $Z^{\prime}$ model, JHEP 03 (2007) 120 [hep-ph/0701107] [INSPIRE].

[40] D. Feldman, Z. Liu and P. Nath, The Stueckelberg $Z^{\prime}$ extension with kinetic mixing and milli-charged dark matter from the hidden sector, Phys. Rev. D 75 (2007) 115001 [hep-ph/0702123] [INSPIRE].

[41] G. Shiu, P. Soler and F. Ye, Millicharged dark matter in quantum gravity and string theory, Phys. Rev. Lett. 110 (2013) 241304 [arXiv:1302.5471] [INSPIRE].

[42] T. Banks and N. Seiberg, Symmetries and strings in field theory and gravity, Phys. Rev. D 83 (2011) 084019 [arXiv:1011.5120] [InSPIRE].

[43] E. Dudas, Y. Mambrini, S. Pokorski and A. Romagnoni, (In)visible $Z^{\prime}$ and dark matter, JHEP 08 (2009) 014 [arXiv:0904.1745] [INSPIRE].

[44] G. Jungman, M. Kamionkowski and K. Griest, Supersymmetric dark matter, Phys. Rept. 267 (1996) 195 [hep-ph/9506380] [InSPIRE].

[45] G. Bertone, D. Hooper and J. Silk, Particle dark matter: evidence, candidates and constraints, Phys. Rept. 405 (2005) 279 [hep-ph/0404175] [INSPIRE].

[46] P. Agrawal, Z. Chacko, C. Kilic and R.K. Mishra, A classification of dark matter candidates with primarily spin-dependent interactions with matter, arXiv:1003.1912 [INSPIRE].

[47] M. Garny, A. Ibarra, M. Pato and S. Vogl, Internal bremsstrahlung signatures in light of direct dark matter searches, arXiv:1306.6342 [INSPIRE].

[48] K. Griest and D. Seckel, Three exceptions in the calculation of relic abundances, Phys. Rev. D 43 (1991) 3191 [INSPIRE].

[49] J. Edsjo and P. Gondolo, Neutralino relic density including coannihilations, Phys. Rev. D 56 (1997) 1879 [hep-ph/9704361] [INSPIRE].

[50] P. Gondolo et al., DarkSUSY: computing supersymmetric dark matter properties numerically, JCAP 07 (2004) 008 [astro-ph/0406204] [INSPIRE].

[51] G. Bélanger, F. Boudjema, A. Pukhov and A. Semenov, Dark matter direct detection rate in a generic model with MicrOMEGAs 2.2, Comput. Phys. Commun. 180 (2009) 747 [arXiv: 0803.2360] [INSPIRE].

[52] XENON100 collaboration, E. Aprile et al., Dark matter results from 225 live days of XENON100 data, Phys. Rev. Lett. 109 (2012) 181301 [arXiv:1207.5988] [INSPIRE].

[53] XENON10 collaboration, J. Angle et al., A search for light dark matter in XENON10 data, Phys. Rev. Lett. 107 (2011) 051301 [arXiv:1104.3088] [INSPIRE]. 
[54] M. Felizardo et al., Final analysis and results of the phase II SIMPLE dark matter search, Phys. Rev. Lett. 108 (2012) 201302 [arXiv:1106.3014] [INSPIRE].

[55] E. Behnke et al., Improved limits on spin-dependent WIMP-proton interactions from a two liter $C_{3} I$ bubble chamber, Phys. Rev. Lett. 106 (2011) 021303 [arXiv: 1008.3518] [INSPIRE].

[56] S. Archambault et al., Dark matter spin-dependent limits for WIMP interactions on F-19 by PICASSO, Phys. Lett. B 682 (2009) 185 [arXiv:0907.0307] [INSPIRE].

[57] XENON100 collaboration, E. Aprile et al., Limits on spin-dependent WIMP-nucleon cross sections from 225 live days of XENON100 data, Phys. Rev. Lett. 111 (2013) 021301 [arXiv: 1301.6620] [INSPIRE].

[58] CDMS collaboration, Z. Ahmed et al., Search for weakly interacting massive particles with the first five-tower data from the cryogenic dark matter search at the Soudan underground laboratory, Phys. Rev. Lett. 102 (2009) 011301 [arXiv:0802.3530] [INSPIRE].

[59] CDMS-II collaboration, Z. Ahmed et al., Results from a low-energy analysis of the CDMS II germanium data, Phys. Rev. Lett. 106 (2011) 131302 [arXiv:1011.2482] [INSPIRE].

[60] R. Bernabei et al., Dark matter investigation by DAMA at Gran Sasso, Int. J. Mod. Phys. A 28 (2013) 1330022 [arXiv:1306.1411] [InSPIRE].

[61] CoGeNT collaboration, C. Aalseth et al., Results from a search for light-mass dark matter with a P-type point contact germanium detector, Phys. Rev. Lett. 106 (2011) 131301 [arXiv: 1002 .4703] [INSPIRE].

[62] G. Angloher et al., Results from $730 \mathrm{~kg}$ days of the CRESST-II dark matter search, Eur. Phys. J. C 72 (2012) 1971 [arXiv:1109.0702] [INSPIRE].

[63] CDMS collaboration, R. Agnese et al., Silicon detector dark matter results from the final exposure of CDMS II, Phys. Rev. Lett. accepted (2013) [arXiv:1304.4279] [INSPIRE].

[64] J.L. Feng, J. Kumar and D. Sanford, Xenophobic dark matter, Phys. Rev. D 88 (2013) 015021 [arXiv: 1306. 2315] [INSPIRE].

[65] CMS collaboration, Search for new physics in the multijets and missing momentum final state in proton-proton collisions at $8 \mathrm{TeV}$, CMS-PAS-SUS-13-012, CERN, Geneva Switzerland (2013).

[66] ATLAS collaboration, Search for squarks and gluinos with the ATLAS detector in final states with jets and missing transverse momentum and $20.3 \mathrm{fb}^{-1}$ of $\sqrt{s}=8 \mathrm{TeV}$ proton-proton collision data, ATLAS-CONF-2013-047, CERN, Geneva Switzerland (2013).

[67] CMS collaboration, Search for supersymmetry in hadronic final states with missing transverse energy using the variables $\alpha_{T}$ and b-quark multiplicity in pp collisions at $8 \mathrm{TeV}$, Eur. Phys. J. C 73 (2013) 2568 [arXiv: 1303.2985] [INSPIRE].

[68] J. Alwall, M. Herquet, F. Maltoni, O. Mattelaer and T. Stelzer, MadGraph 5: going beyond, JHEP 06 (2011) 128 [arXiv:1106.0522] [INSPIRE].

[69] N.D. Christensen and C. Duhr, FeynRules - Feynman rules made easy, Comput. Phys. Commun. 180 (2009) 1614 [arXiv:0806.4194] [INSPIRE].

[70] W. Beenakker, R. Hopker and M. Spira, PROSPINO: a program for the production of supersymmetric particles in next-to-leading order QCD, hep-ph/9611232 [INSPIRE].

[71] T. Sjöstrand, S. Mrenna and P.Z. Skands, A brief introduction to PYTHIA 8.1, Comput. Phys. Commun. 178 (2008) 852 [arXiv:0710.3820] [INSPIRE]. 
[72] M. Cacciari, G.P. Salam and G. Soyez, FastJet user manual, Eur. Phys. J. C 72 (2012) 1896 [arXiv:1111.6097] [INSPIRE].

[73] T. Sjöstrand, S. Mrenna and P.Z. Skands, PYTHIA 6.4 physics and manual, JHEP 05 (2006) 026 [hep-ph/0603175] [INSPIRE].

[74] J.S. Conway, Pretty Good Simulation of high-energy collisions, 090401 release, (2012).

[75] A. Barr, C. Lester and P. Stephens, $m_{T 2}$ : the truth behind the glamour, J. Phys. G 29 (2003) 2343 [hep-ph/0304226] [INSPIRE].

[76] PAMELA collaboration, O. Adriani et al., PAMELA results on the cosmic-ray antiproton flux from $60 \mathrm{MeV}$ to $180 \mathrm{GeV}$ in kinetic energy, Phys. Rev. Lett. 105 (2010) 121101 [arXiv: 1007.0821] [INSPIRE].

[77] S. Chang, R. Edezhath, J. Hutchinson and M. Luty, Effective WIMPs, arXiv:1307.8120 [INSPIRE]. 